\title{
OPEN De novo transcriptome analysis of Chlorella sorokiniana: effect of glucose assimilation, and moderate light intensity
}

\author{
Siti Nor Ani Azaman ${ }^{1,2}$, Darren C. J. Wong ${ }^{3}$, Sheau Wei Tan' ${ }^{4}$, Fatimah M. Yusoff ${ }^{5,6}$,
} Norio Nagao ${ }^{2,7}$ \& Swee Keong Yeap ${ }^{8 \bowtie}$

Chlorella can produce an unusually wide range of metabolites under various nutrient availability, carbon source, and light availability. Glucose, an essential molecule for the growth of microorganisms, also contributes significantly to the metabolism of various metabolic compounds produced by Chlorella. In addition, manipulation of light intensity also induces the formation of secondary metabolites such as pigments, and carotenoids in Chlorella. This study will focus on the effect of glucose addition, and moderate light on the regulation of carotenoid, lipid, starch, and other key metabolic pathways in Chlorella sorokiniana. To gain knowledge about this, we performed transcriptome profiling on C. sorokiniana strain NIES-2168 in response to moderate light stress supplemented with glucose under mixotrophic conditions. A total of $60,982,352$ raw paired-end (PE) reads $100 \mathrm{bp}$ in length was obtained from both normal, and mixotrophic samples of $C$. sorokiniana. After pre-processing, $93.63 \%$ high-quality PE reads were obtained, and 18,310 predicted full-length transcripts were assembled. Differential gene expression showed that a total of 937 , and 1124 genes were upregulated, and downregulated in mixotrophic samples, respectively. Transcriptome analysis revealed that the mixotrophic condition caused upregulation of genes involved in carotenoids production (specifically lutein biosynthesis), fatty acid biosynthesis, TAG accumulation, and the majority of the carbon fixation pathways. Conversely, starch biosynthesis, sucrose biosynthesis, and isoprenoid biosynthesis were downregulated. Novel insights into the pathways that link the enhanced production of valuable metabolites (such as carotenoids in C. sorokiniana) grown under mixotrophic conditions is presented.

Chlorella species is one the most popular microalgae applied in many industries (especially pharmaceuticals, and health) across many Asian countries such as China, Korea, and Japan for hundreds of years ${ }^{1}$. The ability of this species to produce metabolites that possess various health-promoting activities such as antioxidant, antimicrobial, antibacterial, antifungal, anticancer, and antiviral activities make them popular in such industries ${ }^{2-4}$. The potential of producing different pigments by Chlorella species also paves a new way for commercial application of this species, making this system an excellent alternative to current pigment-producing microalgae species. For example, several studies have shown that pigment production was higher in Chlorella compared to other microalgae species ${ }^{5-7}$, and can serve well in commercial applications due to their adaptability to various culture conditions ${ }^{8-10}$.

In an attempt to induce the formation of higher metabolite production such as pigments, and lipid production, manipulation of carbon source, and light intensity were commonly used ${ }^{5-7}$. Carbon sources like glucose, glycerol,

${ }^{1}$ Centre of Foundation Studies for Agricultural Sciences, Universiti Putra Malaysia, Serdang, Selangor, Malaysia. ${ }^{2}$ Aquatic Animal Health and Therapeutics Laboratory (AquaHealth), Institute of Bioscience, Universiti Putra Malaysia, Serdang, Selangor, Malaysia. ${ }^{3}$ Ecology and Evolution, Research School of Biology, The Australian National University, Canberra, ACT 2600, Australia. ${ }^{4}$ Laboratory of Vaccine and Biomolecules (VacBio), Institute of Bioscience, Universiti Putra Malaysia, Serdang, Selangor, Malaysia. ${ }^{5}$ International Institute of Aquaculture and Aquatic Sciences (I-AOUAS), Universiti Putra Malaysia, Port Dickson, Negeri Sembilan, Malaysia. ${ }^{6}$ Department of Aquaculture, Faculty of Agriculture, Universiti Putra Malaysia, Serdang, Selangor, Malaysia. ${ }^{7} 102$ Naname-go, Shinkamigoto-cho, Minami Matsuura-Gun, Nagasaki 857-4214, Japan ${ }^{8} \mathrm{China}$-ASEAN College of Marine Sciences, Xiamen University Malaysia, Sepang, Selangor, Malaysia. ${ }^{\circledR}$ email: skyeap@xmu.edu.my 


\begin{tabular}{|l|l|l|l|l|}
\hline \multirow{2}{*}{ Sample } & \multicolumn{2}{l|}{ Total sequences } & \multicolumn{2}{l|}{ Average length } \\
\cline { 2 - 5 } & Before & After & Before & After \\
\hline Normal replicate 1 & $13,225,251$ & $12,714,292$ & 100 & 79 \\
\hline Normal replicate 2 & $16,604,976$ & $15,073,499$ & 100 & 74 \\
\hline Mixotrophic replicate 1 & $16,690,891$ & $15,817,686$ & 100 & 75 \\
\hline Mixotrophic replicate 2 & $14,461,234$ & $13,492,097$ & 100 & 75 \\
\hline Total & $60,982,352$ & $57,097,573$ & 100 & 75 \\
\hline
\end{tabular}

Table 1. Sequencing throughput, and trimming results for C. sorokiniana RNA-seq data. Two samples: Normal, and Mixotrophic (moderate light stress supplemented with glucose), with three biological replicates of $100 \mathrm{bp}$ reads were generated on the Illumina HiSeq 2000 platform.

or acetate have contributed to increased biomass, and lipid productivity of microalgae ${ }^{11,12}$. Light intensity also influences microalgal growth, and the biochemical composition of cells through the process of photo-acclimation, and photo-adaptation ${ }^{13,14}$. In photo-adaptation, the microalgae change their fatty acid compositions, pigment composition, growth rate, and dark respiration rate $^{13}$. For example, an increase in light intensity led to an increase in chlorophyll a, and other light-harvesting pigments (such as primary carotenoids, chlorophyll b, chlorophyll c, and phycobiliproteins $)^{14}$. On the other hand, when microalgae were exposed to higher light intensity, chlorophyll a, and other pigments directly involved in photosynthesis were reduced, while carotenoids, which serve as photo-protective agents, such as zeaxanthin, $\beta$-carotene, and astaxanthin were found to be increased. However, saturated light intensity, may also disrupt the chloroplast lamellae, and inactivate enzymes involved in carbon dioxide fixation, resulting in photo-inhibition ${ }^{15}$.

Although a lot of studies were reported on the effect of various cultivation condition on metabolite production in microalgae $\mathrm{e}^{16-18}$, a more specific analysis on the effect of genes related to physiological changes were still unpromising. Most were species-specific, and limited to certain treatments only ${ }^{19-21}$. On the other hand, genomic study provides very wide coverage of genetic information on the biological systems of microalgae. For example, Hovde et al. ${ }^{22}$ who studied the genomic characteristics of similar Chlorella species of three different strains showed a significant divergence on their gene content, and nucleotide identity. They also found high variability in transcriptional regulation between the three strains due to distinctive epigenetic machinery. These findings also suggest that each strain had adapted their genomic content according to the new maintained environment. Therefore, individual assessment of different strain is potentially required for understanding their performance in cultivation systems to improve the biomass, and bioproduct yields. In the other genomic report, there are highly conserved genes among the Chlorella species found in the chloroplast providing information that could be applied in microalgal systematics, phylogenetic reconstruction, and biotechnology ${ }^{23}$. In the construction of a microalgal system for commercial application, transcriptomic study could be more efficient, and relatively economical compared to genomic study ${ }^{24}$. It provides an initial, and broad view of molecular, and biochemical mechanisms related to the treatment, and condition used ${ }^{21,25,26}$. Moreover, a growing number of transcriptomes of microalgae were sequenced, assembled, and annotated, providing information on how genes are regulated, and reveal details of the microalgal biology ${ }^{27-30}$.

In our previous study, we examined the biochemical changes, and the morphology of microalgae Chlorella sorokiniana under photoautotrophic, and mixotrophic conditions ${ }^{31}$. We found that glucose, and moderate light intensity treatments contributed to a higher scavenging activity, key changes in pigmentation (i.e.shifts from chlorophyll to carotenoids), and accumulation of lipid bodies compared to the normal light condition. To date, molecular insights into the combinatorial effect of glucose, and light intensity in microalgae, or specifically in Chlorella species is lacking. Thus, this study explored the effect of mixotrophic condition (i.e. moderate light intensity supplemented with $2 \%$ glucose under nutrient-limited condition) on C. sorokiniana using transcriptomics. Several novel findings on the genes involved, and their related pathways, especially the production of secondary metabolites, are highlighted.

\section{Results}

Illumina sequencing, and de novo assembly. The transcriptome of $C$. sorokiniana grown under normal, and mixotrophic conditions generated from RNA with RIN $>7.8$ was sequenced using Illumina sequencing platform. A total of 60,982,352 raw paired-end (PE) reads $100 \mathrm{bp}$ in length were obtained from both normal, and mixotrophic samples of C. sorokiniana. After read trimming, and quality filtering, 57,097,573 (93.63\%) highquality PE reads were obtained (Table 1). A total of 102,643 contigs were successfully assembled using Trinity software $^{32}$. Bonafide transcripts (e.g. complete genes containing both start, and stop codons) were identified using AUGUSTUS resulting in a final assembly of 18,310 full-length contigs (defined as transcripts from here on in). The transcripts ranged from 165 to $16,695 \mathrm{bp}$ in length with the mean length of $1191 \mathrm{bp}$, and an N50 value of 1446 bp were obtained (Table 2). Sequencing data were deposited in Gene Expression Omnibus (GEO) at accession number GSE105427.

Functional annotation. In order to assign functional information, the transcripts were annotated through multiple databases (see "Materials and methods" section). Functional annotation using the NR database showed the highest match, in which $99.8 \%$ (or 18,276 genes) of C. sorokiniana transcripts had succesfull hits (Table 3). When the assembled transcripts were annotated against InterPro, Swiss Prot, Mercator Mapman, and Refseq, 


\begin{tabular}{|l|l|c|}
\hline Subject & Number of reads & Length (bp) \\
\hline Total number of high-quality paired-end reads & $57,097,573$ & \\
\hline Number of contigs & 102,643 & \\
\hline Number of protein-coding transcripts & 18,310 & \\
\hline N50 (bp) & & 1,446 \\
\hline N90 (bp) & & 654 \\
\hline Longest transcript (bp) & & 16,695 \\
\hline
\end{tabular}

Table 2. Summary of de-novo assembly result for C. sorokiniana RNA-seq data.

\begin{tabular}{|l|l|l|}
\hline Public database & No. of annotated genes & \% of annotated genes \\
\hline NR & 18,276 & 99.8 \\
\hline InterPro & 10,019 & 54.7 \\
\hline UniProt/Swiss Prot & 10,259 & 56.0 \\
\hline KO & 6971 & 38.1 \\
\hline GO & 7896 & 43.1 \\
\hline Mapman & 9341 & 51.0 \\
\hline Refseq & 10,187 & 55.6 \\
\hline
\end{tabular}

Table 3. Summary of the functional annotation of the assembled C. sorokiniana transcriptome using different databases.

$50 \%$ to $55 \%$ of $C$. sorokiniana transcripts had matches, whereas, annotation to KEGG, and GO databases produced $38.1 \%$, and $43.1 \%$ matches, respectively.

Based on the NR annotation result, the E-value frequency distribution analysis revealed that $70.3 \%$ of the matched sequences had strong homology with E-value $\leq 1.0 \mathrm{E}-60$, while the remaining $29.7 \%$ fell into the range of $1.0 \mathrm{E}-60$ to $1.0 \mathrm{E}-5$ (Fig. 1). Furthermore, we also observed that all the annotated sequences had a similarity of more than $70 \%$. Based on the homologous species identified among the annotated transcripts, $71.6 \%$ of them matched to Chlorella variabilis, followed by Auxenochlorella protothecoids (5.5\%), Chlamydomonas reinhardtii (3.8\%), Coccomyxa subellipsoidea (1.7\%), and Volvox carteri (1.2\%). About $16.3 \%$ of the transcripts had similarity matches with other types of viridiplantae. In addition, Mercator determined that 9341 transcripts (51\%) had at least one ascribed Mapman annotation.

In the subsequent functional analysis, the functional annotation was done based on the KO (KEGG Orthology) database using KOALA tools (available at KEGG Web site; https://www.kegg.jp) that assign the KO identifiers (K numbers) to the transcript by BLAST, and GHOSTX searchers. In this study, of the 18,310 transcripts that aligned with $\mathrm{KO}$ database, only 6971 transcripts (38.1\%) were annotated, and had significant matches to different functional categories (Fig. 2). The highest number of genes identified was from the genetic information processing category, which were 3007 genes (43\%). The second highest functional categories of transcripts identified were carbohydrate metabolism ( 637 genes), followed by environmental information processing (563 genes), amino acid metabolism (377 genes), lipid metabolism (329 genes), energy metabolism (304 genes), cellular processes ( 311 genes), and metabolism of cofactor, and vitamins (231 genes). The remaining $17 \%$ of KEGG annotated transcripts were from other categories.

Under pathway reconstruction of $\mathrm{KO}$, each transcript from different functional categories was further elucidated into different metabolic pathways. All the metabolic pathways were then divided into four main pathway modules such as metabolism, genetic information processing, environmental information processing, and cellular processes (Fig. 3). According to this pathway assignment, 5018 transcripts (27.4\% of all transcripts) were classified into 23 KEGG pathways. From the annotation result, the transcripts coding for all the genes related to the major metabolic pathways in C. sorokiniana were identified. The completeness of these reconstructed pathways indicates that the gene function assignments were biologically meaningful, and the EC number(s) has been correctly assigned to the annotated sequences (Table 4). Other KEGG pathways with multiple transcript hits encoding for a nearly complete pathway include nucleotide, and amino acid metabolism (such as serine, and threonine biosynthesis, lysine biosynthesis, and histidine biosynthesis), and cofactor, and vitamin biosynthesis (such as shikimate pathway, phenylalanine biosynthesis, coenzyme A biosynthesis, and biotin biosynthesis).

Differential expression analysis. Differential expression analysis between normal, and mixotrophic conditions revealed 2061 differentially expressed transcripts among the treatments, of which 937, and 1124 were upregulated, and downregulated in mixotrophic samples, respectively (Supplementary Data 1; Supplementary Figure S1, and S2). The biological pathways that were significantly affected (Adj. $P<0.05$ ) by mixotrophic conditions are summarised in Fig. 4, and briefly described below. Detail information of genes involved in different metabolic pathways can be found in Supplementary Figures, and Tables. 
a

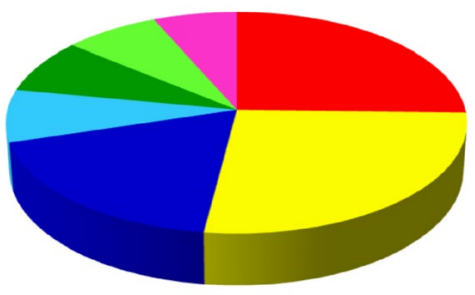

0

- $1 \mathrm{e}-100$ to $1 \mathrm{e}-60$

- $1 \mathrm{e}-45$ to $1 \mathrm{e}-30$

- $1 \mathrm{e}-15$ to $1 \mathrm{e}-5$
0 to $1 \mathrm{e}-100$

$1 e-60$ to $1 e-45$ b

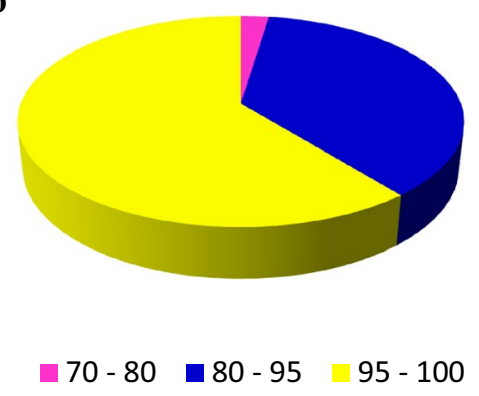

Chlorella variabilis

c

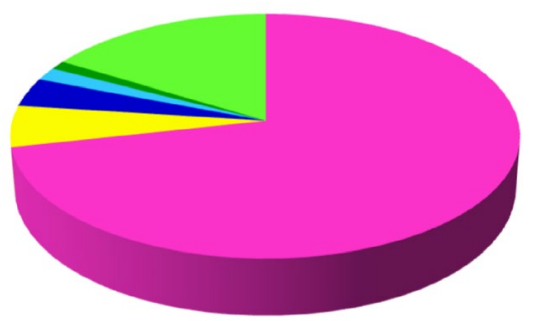

Auxenochlorella protothecoids

- Chlamydomonas reinhardtii

- Coccomyxa subellipsoidea

Volvox carteri

Others

Figure 1. Transcript homology searches against the nr database. (a) The proportional frequency of the E-value distribution. (b) The proportional frequency of the sequence similarity distribution. (c) The proportional species distribution of C. sorokiniana transcriptome among other viridiplantae.

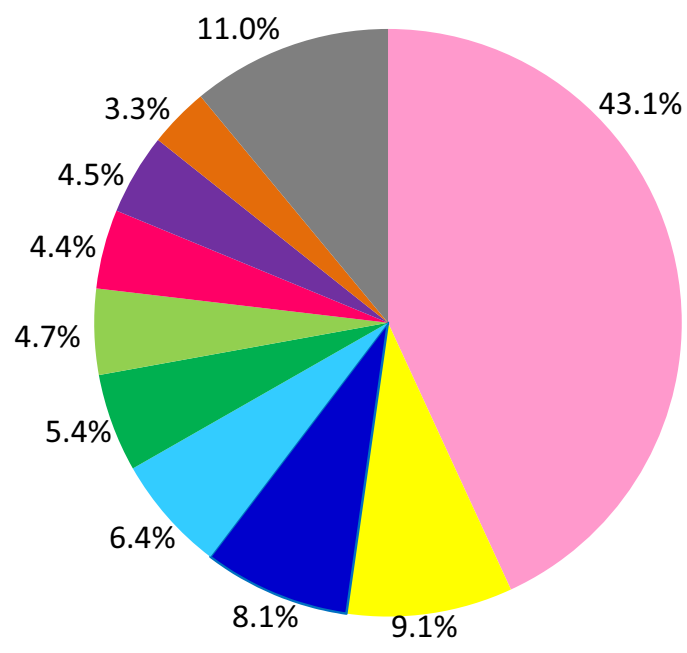

\author{
Genetic Information Processing \\ Carbohydrate metabolism \\ Environmental Information Processing \\ - Unclassified \\ Amino acid metabolism \\ - Lipid metabolism \\ Energy metabolism \\ - Cellular Processes \\ Metabolism of cofactors and vitamins \\ - Other categories
}

Figure 2. Annotation of C. sorokiniana transcripts based on functional categories KO database using KOALA tools at KEGG Web site ${ }^{58}$ (https://www.kegg.jp/blastkoala/).

Secondary metabolism and carotenoids biosynthesis. Biosynthesis of secondary metabolites including carotenoids, phenols, and other isoprenoids originated from two pathways: mevalonate pathway, and non-mevalonate (or MEP) pathway. It is well known that carotenoids biosynthesis in C. sorokiniana is primarily biosynthesized via the plastid-localized non-mevalonate (or MEP/DOXP) pathway. All the key enzymes involved in the synthesis of important precursors for pigments biosynthesis, which are isopentenyl pyrophosphate (IPP), and dimethylallyl pyrophosphate (DMAPP) were identified (Supplementary Figure S3). The nonidentified enzyme for mevalonate kinase (MVK, EC 2.7.1.36) confirmed that this pathway occurs in the plastids, 


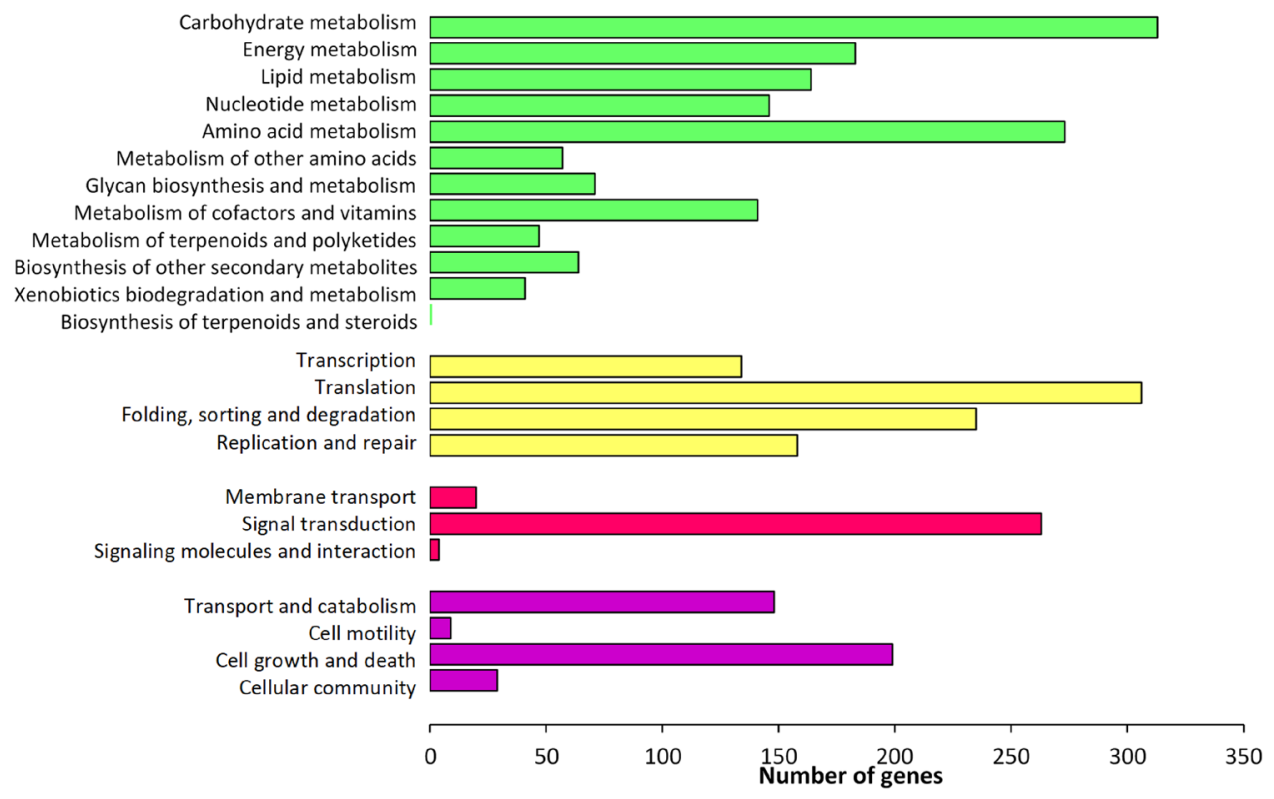

Figure 3. Functional classification, and pathway assignment of unigenes by KEGG Orthology (KO). The results are summarized in five main pathways modules: Metabolism (green); Genetic Information Processing (yellow); Environmental Information Processing (red); and Cellular Processes (purple). The y-axis indicates the name of the KEGG metabolic pathways. The $\mathrm{x}$-axis indicates the number of genes annotated under the pathway.

\begin{tabular}{|l|l|l|}
\hline Pathway & Number of genes found & Number of known genes \\
\hline Reductive pentose phosphate (Calvin-Bensen cycle) & 11 & 12 \\
\hline Glycolysis/gluconeogenesis & 10 & 10 \\
\hline Citrate cycle (TCA cycle) & 9 & 10 \\
\hline Fatty acid biosynthesis & 6 & 6 \\
\hline Triacylglycerol (TAG) biosynthesis & 4 & 4 \\
\hline Starch biosynthesis & 4 & 4 \\
\hline Isoprenoid biosynthesis (MEP pathway) & 7 & 7 \\
\hline
\end{tabular}

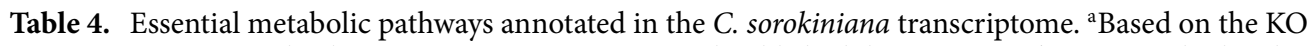
annotation in KEGG database using BLASTKOALA, and published data in C. sorokiniana, and related green $\operatorname{algae}^{21,44,65}$.

not in the cytoplasm, which is similar to the other photosynthetic green algae ${ }^{33}$. Our transcriptome analysis revealed that the transcripts encoding genes potentially responsible for carotenoid production was generally upregulated when moderate light intensity, and glucose addition were applied to the C. sorokiniana culture (Table S1). This was evidenced by the upregulation of transcripts encoding 2-C-methyl-D-erythritol 4-phosphate cytidylyltransferase (CMS) or IspD; the gene that responsible for the production of an intermediate molecule from MEP to produce isopentenyl diphosphate (IPP), and dimethylallyl diphosphate (DMAPP). In the downstream step, lutein production was also upregulated (Fig. 4). This was evidenced by the upregulation of carotenoids lycopene epsilon cyclase, and $\varepsilon$-ring hydroxylase (Table S2). These two genes are required to cyclise the hydrocarbon chain, and hydroxylate the carotene rings to generate xanthophylls such as lutein, and zeaxanthin ${ }^{34}$. On the other hand, two genes related to the production of $\beta$-carotene, and astaxanthin were downregulated; they were phytoene dehydrogenase, and carotenoid cleavage dioxygenase ${ }^{35}$. Downregulation of these two genes showed that the mixotrophic condition was favourable forlutein production but not towards the biosynthesis of other carotenoids, and apocarotenoids (such as vitamin A, retinol, and abscisic acid hormone) ${ }^{36}$.

In the case of isoprenoid metabolism (see monoterpene biosynthesis Fig. 4), the downregulation of HMGS, the gene that converts cytosolic acetyl-CoA into HMG-CoA via the mevalonate pathways, is indicative of a general downregulation of the isoprenoid biosynthesis. Further downstream of this pathway showed downregulation of several genes such as those related with phenylpropanoid (lignin) biosynthesis which is cinnamyl alcohol dehydrogenase (CAD); gene related with tocopherol (vitamin E) biosynthesis which is MSBQ methyltransferase; and those related with monoterpenes biosynthesis which isogeranyl diphosphate (GPP) synthase (Supplementary Data 1, and Figure S4) ${ }^{37}$. 


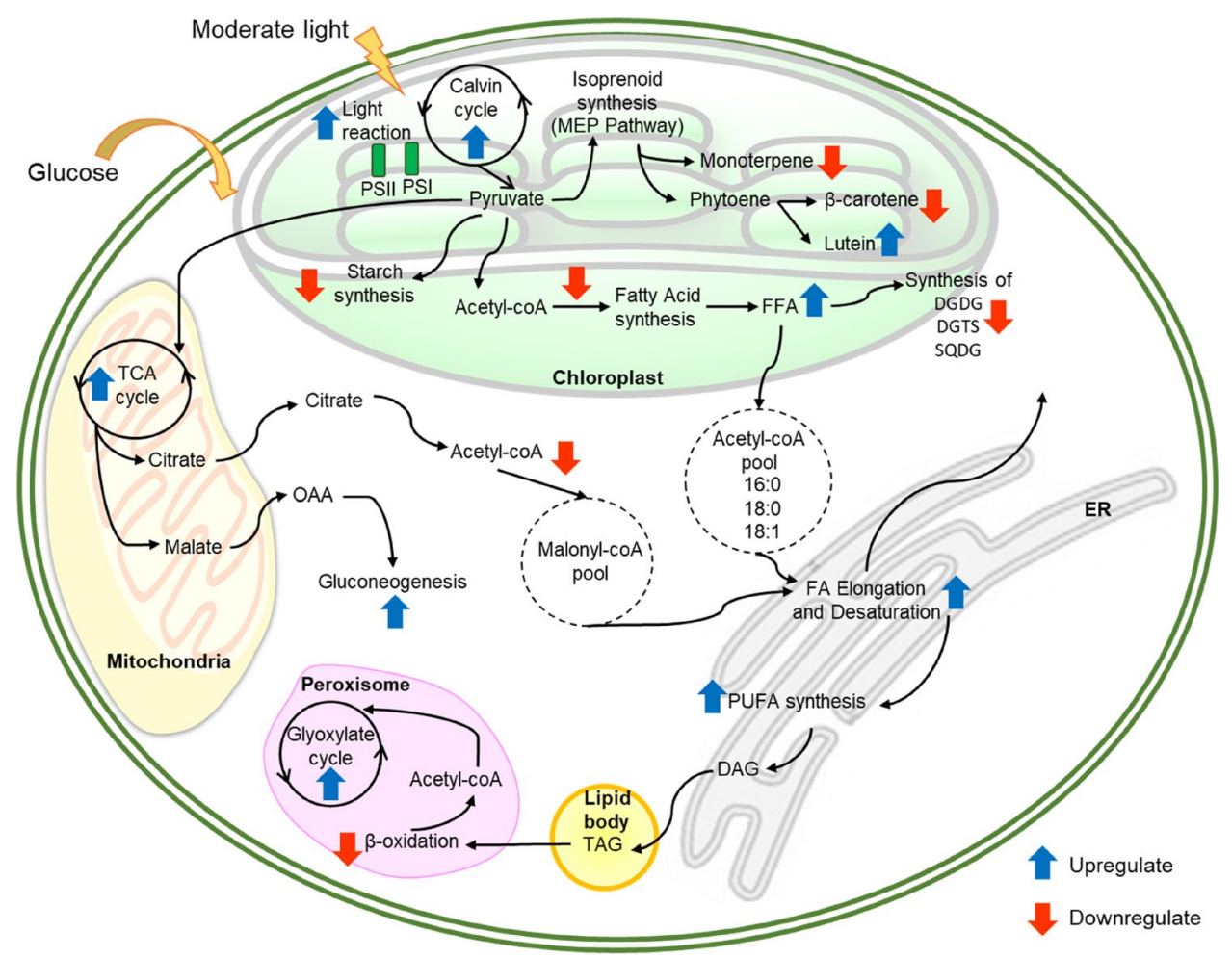

Figure 4. Overview of key metabolic pathways responsive to moderate light stress under nutrient-limiting conditions in C. sorokiniana Upregulation or downregulation of mRNA expression under stress condition based on transcriptomic analysis are indicated with blue upward arrows, and red downward arrows, respectively. PSI photosystem I, PSII photosystem II, FFA free fatty acid, OAA oxaloacetate, FA fatty acid, PUFA polyunsaturated fatty acid, DGDG digalactosyl diacylglycerol, DGTS diacylglyceryl N,N,N-trimethyl homoserine, SQDG sulfoquinovosyl diacylglycerol, TCA tricarboxylic acid, ER endoplasmic reticulum.

Fatty acid biosynthesis, and lipid metabolism. The fatty acid biosynthesis in microalgae occurs in two subcellular components; first, de novo synthesis of fatty acids in plastids, and second, conversion into long-chain fatty acid occurs in the endoplasmic reticulum ${ }^{38}$. All transcripts encoding genes related to fatty acid biosynthesis, and TAG synthesis were detected in this C. sorokiniana transcriptome (Table 4). Under mixotrophic condition imposed in this study, the first step of fatty acid synthesis was downregulated, as detected by the downregulation of acetyl coA carboxylation reaction of both types of acetyl coA carboxylase (homomeric, and heteromeric) transcripts (Table S3). The plastidial acetyl coA carboxylase transcripts, whose products form a heteromeric multi-subunit enzyme complex that contains biotin carboxylase activity, whereas the cytosolic acetyl coA carboxylase is a homomeric multifunctional protein that does not contain biotin carboxylase activity ${ }^{39}$.

However, the subsequent steps in the lipid metabolism such as desaturation, and elongation processes were upregulated (Table S4-S6). This is evident by the upregulation of transcripts encoding acetyl coA ACP transacylase (FabH); ketoacyl ACP synthase (FabB, and FabF); oxoacyl ACP reductase (FabG); steryl-ACP desaturase (DESA1); stearoyl-coA desaturase (SCD or desC); and omega-6 desaturase (FAD2 or desA) in biosynthesis, and desaturation processes. DESA1, and desA responsible for desaturation step, and further exported to the cytosol into the acyl-coA, and acyl-lipid pools in the cytoplasm ${ }^{38-40}$. Meanwhile, elongation in endoplasmic reticulum showed upregulated as detected by ketoacyl coA synthase (KCS), acyl-coA reductase (FAR), very-long chain (3R)-hydroxyacyl-coA dehydratase (PAS1), and very long chain enoyl-coA reductase.

In the case of glycerolipid, and triacylglycerol (TAG) metabolism, most transcripts detected were downregulated, including, glycerol-3-phosphate $O$-acyltransferase (GPAT), lysophospholipid acyltransferase (LPLAT), TAG lipase (TagL), and diacylglycerol kinase (DGK). Several other transcripts encoding genes involved in the synthesis of membrane lipids especially thylakoid membrane such as UDP-sulfoquinovose synthase (SQD1), sulfolipid synthase (SQD2), phosphatidate cytidylyltransferase (CDS), cyclopropane-fatty-acyl-phospholipid synthase (CFAS), and phosphatidylcholinesterol O-acyltransferase (LCAT) were also downregulated (Table S7). All these transcripts involved in the formation of membrane components such as digalactosyl diacylglycerol (DGDG), diacylglyceryl N,N,N-trimethyl homoserine (DGTS), and sulfoquinovosyl diacylglycerol (SQDG) (Fig. 4).

Carbohydrate metabolism. Generally, most carbohydrate metabolic pathways (such as glycolysis, starch, and sucrose metabolisms) were downregulated (Table S9-S11). However, some transcripts involved in the glycolysis process were upregulated. For example, aldolase (ALDO), triose phosphate dehydrogenase (GAPDH), and 


\begin{tabular}{|c|c|c|c|c|c|}
\hline Gene symbol & Target gene & Forward/reverse primers $\left(5^{\prime}-3^{\prime}\right)$ & Product size (bp) & Efficiency value (\%) & $\mathbf{R}^{2}$ \\
\hline \multirow[t]{2}{*}{ RPL } & \multirow[t]{2}{*}{ Ribosomal protein L19 } & $\begin{array}{l}\text { GTCTGGCTGGACCCCAAT } \\
\text { GA }\end{array}$ & \multirow[t]{2}{*}{143} & \multirow[t]{2}{*}{90.4} & \multirow[t]{2}{*}{0.999} \\
\hline & & GCCTCTGCTGCGGTGC & & & \\
\hline \multirow[b]{2}{*}{ A-tub } & \multirow[b]{2}{*}{ Alpha-tubulin } & CACAGTTTACCCGTCTCCCC & \multirow[b]{2}{*}{185} & \multirow[b]{2}{*}{97.6} & \multirow[b]{2}{*}{0.996} \\
\hline & & $\begin{array}{l}\text { TCAGCCGGTTAAGGTTGG } \\
\text { TG }\end{array}$ & & & \\
\hline \multirow{2}{*}{ PCNA } & \multirow{2}{*}{ Proliferating cell nuclear antigen } & $\begin{array}{l}\text { TGGGCCTGAACCTGAACA } \\
\text { AC }\end{array}$ & \multirow{2}{*}{301} & \multirow{2}{*}{90.3} & \multirow{2}{*}{0.987} \\
\hline & & TTGATGCCCTCCTTCGTCAC & & & \\
\hline \multirow[b]{2}{*}{ DESA 1} & \multirow[b]{2}{*}{ Stearyl-ACP desaturase } & TGCTGGGTCGCTTCCTCTAC & \multirow[b]{2}{*}{397} & \multirow[b]{2}{*}{94.3} & \multirow[b]{2}{*}{0.995} \\
\hline & & $\begin{array}{l}\text { CCGTTGTTCAGCTCCGAG } \\
\text { TG }\end{array}$ & & & \\
\hline \multirow[t]{2}{*}{$\mathrm{MDH}$} & \multirow{2}{*}{ Malate dehydrogenase } & $\begin{array}{l}\text { TCACCAAGGAGGAGATTG } \\
\text { AG }\end{array}$ & \multirow[t]{2}{*}{342} & \multirow[t]{2}{*}{105.3} & \multirow[t]{2}{*}{0.989} \\
\hline & & CGCCTGTCTTAATGTTGGTC & & & \\
\hline \multirow{2}{*}{ IspD } & \multirow{2}{*}{$\begin{array}{l}\text { 2-C-methyl-D-erythritol 4-phos- } \\
\text { phate cytidylyltransferase }\end{array}$} & \begin{tabular}{|l} 
CTGGTGGCAATTCACGACTC \\
\end{tabular} & \multirow{2}{*}{263} & \multirow{2}{*}{99.3} & \multirow{2}{*}{0.981} \\
\hline & & TCAGTGACCTCCAGCTTCTC & & & \\
\hline \multirow{2}{*}{ ACACA } & \multirow{2}{*}{ Acetyl Coa carboxylase } & $\begin{array}{l}\text { GCATGGCCGACCAGTTTG } \\
\text { TG }\end{array}$ & \multirow{2}{*}{347} & \multirow{2}{*}{94.1} & \multirow{2}{*}{0.966} \\
\hline & & $\begin{array}{l}\text { CCGGTCGTAGACATCAGG } \\
\text { TG }\end{array}$ & & & \\
\hline \multirow{2}{*}{ pfkA } & Phosphofruktokinase & CAAGCCCATCACCCTTACCC & 298 & 915 & 0991 \\
\hline & Phosphofruktokınase & CTCCACAGCCGTCTCAAACC & 298 & (1.J & 0.90 \\
\hline & & $\begin{array}{l}\text { GCTGCTGGAACACCAGAT } \\
\text { GC }\end{array}$ & & & \\
\hline TagL & TAG lipase & \begin{tabular}{|l|} 
GGCGCTGTCAGGTAGTTG \\
AG \\
\end{tabular} & 357 & 105.6 & 0.965 \\
\hline $\operatorname{glg} B$ & 1,4-glucan branching enzyme & $\begin{array}{l}\text { GAGCGCGATGACTTTGGC } \\
\text { AC }\end{array}$ & 358 & 98.2 & 0.948 \\
\hline & & TGGCGTTGTAGCCCAGCTTC & & & \\
\hline
\end{tabular}

Table 5. Housekeeping, and target genes, and their primers used for RT-qPCR.

phosphoglycerate kinase (PGK) showed upregulation. Whilst, the other transcript in all three pathways showed downregulation.

Validation of gene expression through quantitative real-time PCR. Quantitative real-time PCR (RT-qPCR) was used to validate 7 differentially expressed genes identified by RNA-seq. The genes were chosen from each of the important pathways in the transcriptomic analysis of RNA-seq data. For example, stearylACP desaturase (DESA1) from desaturation of fatty acid, malate dehydrogenase (MDH) from TCA cycle, 2-C-methyl-D-erythritol 4-phosphate cytidylyltransferase (IspD) from MEP pathway, Acetyl-Coa carboxylase (ACACA) from fatty acid biosynthesis pathway, TAG lipase (TagL) from TAG biosynthesis pathway, 1,4-glucan branching enzyme (glgB) from starch biosynthesis pathway, and phosphofruktokinase (pfkA) from glycolysis pathway. The primers used for each target genes, and selected housekeeping genes with their efficiency, and $\mathrm{R}^{2}$ data are shown in Table 5. In RNA-seq sequencing results, the expression levels of DESA1, MDH, and IspD were significantly upregulated, whilst the expression of ACACA, TagL, glgB, and pfkA were significantly downregulated in mixotrophic conditions. The gene expression profile based on RT-qPCR was highly correlated $(\mathrm{R}=0.916)$ with RNA-seq confirming the reliability, and validity of the RNA-seq technique (Fig. 5).

\section{Discussion}

Chlorella sorokiniana can grow in various growth conditions making them a target organism for sustainable metabolite production. Findings from several microalgae have previously shown that mixotrophic conditions are more desirable to achieve both higher growth rates, and biomass yields (and even lipid content) ${ }^{41,42}$. In the present study, we induced the production of carotenoids, and lipids production through glucose assimilation, and light mixotrophic condition, and investigated the expression of genes involved in carotenoids, and lipid accumulation at the transcriptome level for the very first time. However, this transcriptomic analysis is not a substitute for detailed gene, and pathway studies, but it provides a broad overview of the important metabolic process from which to efficiently build a hypothesis that can guide future detailed studies on improving the carotenoid production, and lipid accumulation in this and others microalgae.

In the case of isoprenoids biosynthesis, there were two pathways responsible for the production of these biomolecules; the MVA, and MEP pathway. The incomplete genes for MVA pathway in this C. sorokiniana transcriptome suggest that the production of isoprenoids was dependent on MEP pathway that exists in the plastid. In our study, we found that homologs of the MVA pathway genes such as hydroxymethyl-glutaryl-CoA (HMGcoA) synthase (HMGS) exist in C. sorokinina. Conversely, homologs encoding hydroxymethyl-glutaryl-CoA 


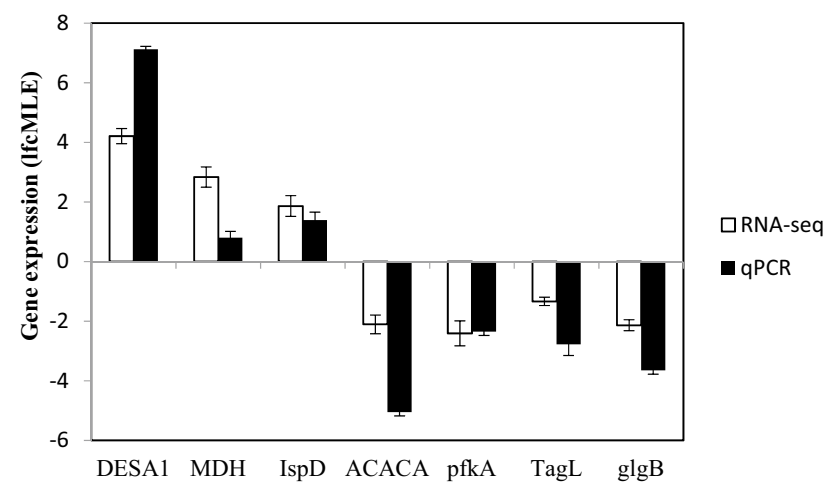

Figure 5. RT-qPCR validation of the gene expression patterns. The white bars represent the changes in the transcript from RNA-seq, and the black bars represent the relative expression level estimated by RT-qPCR. Whisker in each bar represents standard error.

reductase (HMGR), mevalonate kinase (MVK), phosphomevalonate kinase (PMK), and diphosphomevalonate decarboxylase (MVD) were absent. These results were proportional to the results of studies on Ulva prolifera ${ }^{30}$, Porphyra umbilicalis ${ }^{29}$, and Chlorella zofingiensis ${ }^{40}$. From the findings, the mixotrophic condition used in this study reduced the expression of sterols, sesquiterpenes, and triterpenes. These isoprenoids are secondary metabolites responsible to produce various bioactive compounds (such as antioxidant, antibacterial, antifungal, etc.) in microalgae ${ }^{40}$. The downregulation of the genes related to isoprenoids biosynthesis might explain our previous results regarding the level of phenolic compound, and their lowered antioxidant activity compared to normal growth condition ${ }^{31}$.

Meanwhile, for carotenoids biosynthesis located in the plastids showed that under mixotrophic condition lutein production was more favorable compared to the other carotenoids production. Our result was similar to the study done by Xiao et al. ${ }^{18}$, which they found two $\beta$-ring hydroxylases were upregulated in Auxenochlorella protothecoides culture that produced the highest lutein production. There are two routes leading to lutein production from $\alpha$-carotene: $\beta$-ring hydroxylation to zeinoxanthin followed by $\varepsilon$-ring hydroxylation to lutein or $\varepsilon$-ring hydroxylation to $\alpha$-cryptoxanthin, followed by $\beta$-ring hydroxylation to lutein ${ }^{43}$. In our transcriptome data, the second step after $\beta$-ring hydroxylation which is $\varepsilon$-ring hydroxylase was upregulated leading to lutein production was similarly found by Xiao et al. ${ }^{18}$. In terms of cultivation strategies, they used a two-step transition process from heterotrophic to photoautotrophic condition to increase lutein production-i.e. glucose as the carbon source in heterotrophic growth followed by illumination. The other study that used acetate as the carbon source in the mixotrophic growth of C. sorokiniana, however, their transcriptomic result obtained did not show any genes related to carotenoid production, either lutein or astaxanthin, being differentially expressed ${ }^{44}$. This indicates that glucose shows better results for lutein production compared to acetate. One study has also shown that glucose was the best carbon source for mixotrophic growth of all the Chlorella strains for increasing microalgal biomass, followed by glycerol, sodium acetate, and sucrose ${ }^{12}$. Furthermore, although genes related to the other carotenoid production such as $\beta$-carotene, and astaxanthin were detected in this C. sorokinina transcriptome, the mixotrophic condition was not suitable for their production. Thus, if pigments (such as $\beta$-carotene, and astaxanthin) are needed to be produced from this microalgae, different induction strategies will need to be ascertained.

In lipid metabolism, the downregulation of the first step in fatty acid synthesis followed by the upregulation of genes downstream of fatty acid synthesis, including elongation, and desaturation, showed contradictory results from one earlier study ${ }^{21}$. However, this pattern of regulation showed feedback inhibition of fatty acid synthesis like proposed by Andre et al. ${ }^{45}$. Our result was similar to Sirikhachornkit et al ${ }^{46}$ who found the concomitant accumulation of TAG with downregulation of TAG, and DAG lipase genes under nitrogen deficiencies in $S$. acutus. Thus, these findings may provide a potential target for designing strategies for increasing fatty acid synthesis in microalgae. Meanwhile, the changes in the membrane lipid metabolism were due to the effect of carbon partitioning during mixotrophic conditions. This was discussed in detail by Alboresi et al. ${ }^{47}$, and Han et al. ${ }^{48}$ who investigated the effect of light, and nitrogen deprivation on Nannochloropsis sp. cultures using a combination of omics approach. In their studies, they found that chloroplast polar lipids were decreased, while the other membrane components were increased. This happened due to the accumulation of lipid accompanied by the regulation of inorganic phosphate transport across the chloroplast membranes, and tuning the carbon metabolic allocation between cell compartments such as cytoplasm, mitochondrion, and endoplasmic reticulum. Furthermore, the assimilation of glucose during mixotrophic conditions assisted the accumulation of lipids where the carbon skeleton from glycolysis, gluconeogenesis, and starch degradation might be directly channeled into fatty acids synthesis. The activities of enzymes involved in the starch synthesis pathway were down-regulated in this study supporting the hypothesis of starch to lipid shift under stress condition ${ }^{49}$. Since both synthesis of starch, and TAG share carbon precursors, blocking the starch synthesis can shift the carbon flux towards fatty acid, and TAG accumulation. Several other studies in different microalgae such as C. reinhardtii ${ }^{50}$, Dunaliella tertiolecta $a^{51}$, and Scenedesmus obliquus ${ }^{52}$, which have mutations that affected starch accumulation showed an increase in total lipid, and TAG contents under nitrogen deficiencies. In this study, lipids were stored in the form of polyunsaturated 
fatty acids which was similarly found by other researchers that also studied the effect of mixotrophic condition for biofuel production ${ }^{53}$. Many studies also reported that the microalgae would only produce either lipids or starch in order to increase the specific product yields. This was also seen in other microalgae such as Chlamydomonas reinhardtii, and Miracritinum pusillum ${ }^{5-56}$. On the other hand, repressing the $\beta$-oxidation is a clear strategy for maintaining a higher concentration of fatty acid within cells.

Transcriptomic analysis of C. sorokiniana under mixotrophic conditions enabled the exploration of a broad diversity of genes, and pathways. From the analysis, 18,310 assembled transcripts were obtained. The functional annotation, and classification of these transcripts provided a better understanding of C. sorokiniana transcriptome, and its molecular basis under mixotrophic conditions where glucose was added, and moderate light intensity were used. The findings point to several molecular mechanisms that potentially drive the overproduction of high-value metabolites such as carotenoids especially for lutein, unsaturated fatty acids synthesis, TAG accumulation. Meanwhile, the mixotrophic condition could repress the starch synthesis, sucrose synthesis, and isoprenoid biosynthesis. The transcriptome analysis supported the previous biochemical and morphological findings of C. sorokiniana cultured under photoautotrophic and mixotrophic conditions ${ }^{31}$.

\section{Materials and methods}

Strain. The Chlorella sorokiniana (NIES-2168) used in this study was obtained from the Marine Biotechnology Lab at the Faculty of Agriculture, Universiti Putra Malaysia, which had originally obtained from NIES (National Institute of Environmental Studies, Japan).

Experimental design. The microalgae were cultured in Bold's Basal Medium (BBM) (PhytoTechnology Laboratory ${ }^{\oplus}$, USA). The stock medium was diluted from $50 \times$ to $1 \times$ using sterile distilled water, and adjusted to $\mathrm{pH} 6.6$ using $1 \mathrm{M} \mathrm{NaOH}$. Sterilisation was performed by autoclaving for $20 \mathrm{~min}$ at $121^{\circ} \mathrm{C}$. At the beginning of microalgae pre-culture preparation, the microalgae were first inoculated with $10 \%$ ( $\mathrm{vol} / \mathrm{vol}$ ) of an exponentially growing culture, and allow to grow under continuous light with an intensity of approximately $10 \mu$ mol photons $\mathrm{m}^{-1} \mathrm{~s}^{-1}$ with a shaking speed of $30 \mathrm{rpm}$ at $27^{\circ} \mathrm{C}$. These pre-culture microalgae were allowed to grow until midlogarithmic phase at day 15 , which produce approximately $2.5-3.0 \times 10^{6}$ cells $/ \mathrm{mL}$. Then, the culture was divided into two flasks for normal, and mixotrophic conditions respectively. Each of the flasks contained $100 \mathrm{~mL}$ of $2 \times 10^{6}$ cells $/ \mathrm{mL}$ of $C$. sorokiniana culture. Cultures for the normal condition was allowed to grow under the same condition as pre-culture, whereas culture for mixotrophic condition was allowed to grow under moderate light intensity $\left(100 \mu \mathrm{mol}\right.$ photons $\left.\mathrm{m}^{-1} \mathrm{~s}^{-1}\right)$, and supplemented with $2 \%$ glucose $\mathrm{e}^{31}$. Both cultures were allowed to continue growing for 7 days. The experiments were conducted in a shaking incubator, and a conical flask was used as the growth chamber. A white fluorescence light source was located above the cultures. All experiments were repeated independently in triplicates. The microalgae were harvested by separating the pellet from the medium by centrifugation at $10,000 \mathrm{rpm}$ for $10 \mathrm{~min}$. The pellet was then flash-frozen using liquid nitrogen, and stored at $-20^{\circ} \mathrm{C}$ prior to use.

RNA extraction. Total RNA was extracted using TRIzol method. Briefly, $100 \mathrm{mg}$ of frozen tissue was ground using a prechilled mortar, and pestle into a fine powder. $1 \mathrm{~mL}$ TRIzol reagent (Invitrogen, California) was added to the ground tissue, and homogenisation continued until no visible debri remains. The homogenised samples were incubated at room temperature for $5 \mathrm{~min}$. Chloroform with 0.2 times the volume of Trizol solution was added to the solution, and the mixture was vortexed vigorously for about $15 \mathrm{~s}$, and incubated at room temperature for 2 to $3 \mathrm{~min}$. After centrifugation, the aqueous layer was transferred to a column of RNeasy from a mini RNA isolation kit (Qiagen, Germany) for further purification. The residual DNA was eliminated by performing a column DNase digestion at $37^{\circ} \mathrm{C}$ for $30 \mathrm{~min}$. The integrity of the extracted RNA was determined by gel electrophoresis, and its concentration was measured using a biospectrometer (Eppendorf, Germany).

Library preparation, and Illumina sequencing. The library for RNA-sequencing was prepared using NEBNext Ultra Directional RNA Library Prep kit for Illumina from New England Biolabs (NEB, UK) according to the manufacturer's protocol. The molarity, and libraries sizes were assessed on an Agilent 2100 Bioanalyzer (Agilent Technologies, Germany). Sequencing of 100 bp paired-end reads was performed on the Illumina HiSeq 2000 at HIR Central Lab High End Instrument, University of Malaya, Malaysia.

De novo transcriptome assembly, and annotation. Filtered paired-end reads of each sample were pooled, separated into their respective orientation, and collapsed further to retain only the unique non-redundant reads using the FASTX-toolkit (https://hannonlab.cshl.edu/fastx_toolkit/). The transcriptome de novo assembly was performed using Trinity (version 2.0.6) with default settings ${ }^{32}$. Gene predictions, and proteincoding potential of the de novo assembled transcripts were determined using AUGUSTUS (version 2.5.5) ${ }^{57}$. To obtain an accurate gene model, the Chlamydomonas reinhardtii sequence (genes, and proteins), and gene structure was used to train AUGUSTUS. A comprehensive annotation of the protein-coding gene, and protein repertoire were performed using BLASTN, and BLASTX, respectively against the NCBI non-redundant, SwissProt (and UniProt), Refseq, KEGG (Kyoto Encyclopedia of Genes, and Genomes) ${ }^{58}$, and GO (Gene Ontology) databases using a threshold of E-value $<10^{-5}$ to define significant hits, and for ascribing potential gene function. Functional MapMan BIN categories were ascribed to predicted genes using the Mercator ${ }^{59}$. Default parameters were used with additional 'CHLAMY', and 'IPR' settings enabled. 
Differential gene expression, and functional enrichment analysis. The alignment of filtered paired-end reads against the predicted protein-coding genes was performed using Subread aligner ${ }^{60}$, and counting of reads (read summarization) was performed with Feature Counts ${ }^{61}$ with default settings in R software ${ }^{62}$. Differential gene expression analysis was carried out using DESeq $2^{63}$, and genes were defined as significantly different between treatment conditions, and controls at a false discovery rate $(F D R)<0.05$. Transcript abundance was estimated using DESeq2 using the variance stabilization normalization (VST). Enrichment of MapMan BINs categories was determined by Fisher's exact test adjusted with Bonferonni correction for multiple testing correction using the Corto tool ${ }^{64}$. MapMan BIN categories were deemed significantly enriched at an adjusted $P$ value $<0.05$.

Quantitative real-time PCR (RT-qPCR) validation. Quantitative real-time PCR (RT-qPCR) was carried out to validate the results of the RNA-seq. Total RNA was extracted using TRIzol method as described for RNA extraction. The reverse transcription step for the preparation of the cDNA library was performed using SensiFAST cDNA Synthesis Kit (Bioline, UK) according to the manufacturer's instructions. Three-hundred nanograms of the RNA template from each sample were converted into cDNA in $20 \mu \mathrm{L}$. The control was prepared by using a similar amount of total RNA that was subjected to the cDNA synthesis reaction without the presence of reverse transcriptase. A $300 \mathrm{ng}$ cDNA template pool produced from the reverse transcription reaction was subsequently used to assess housekeeping genes, and target genes transcript levels in real-time PCR assays. The information of primers used for housekeeping genes, and target genes are presented in Table 5.

\section{Data availability}

The authors declare that all the data in this manuscript are available.

Received: 8 June 2020; Accepted: 1 October 2020

Published online: 15 October 2020

\section{References}

1. Priyadarshani, I. \& Rath, B. Commercial and industrial applications of micro algae-a review. J. Algal Biomass Utili. 3, 89-100 (2012).

2. Borowitzka, M. A. Microalgae as a sources of pharmaceuticals and other biologically active compounds. J. Appl. Phycol. 7, 3-15 (1995).

3. Mayer, A. M. \& Hamann, M. T. Marine pharmacology in 2001-2002: marine compounds with anthelmintic, antibacterial, anticoagulant, antidiabetic, antifungal, anti-inflammatory, antimalarial, antiplatelet, antiprotozoal, antituberculosis, and antiviral activities; affecting the cardiovascular, immune and nervous systems and other miscellaneous mechanisms of action. Comp. Biochem. Physiol. C Toxicol. Pharmacol. 140, 265-286 (2005).

4. Mostafa, S. S. Microalgal biotechnology: prospects and applications. INTECH Open Access Publisher. Retrieved from https://www. intechopen.com/books/plant-science on 4 September 2019 (2012).

5. Del Campo, J. A. et al. Carotenoid content of chlorophycean microalgae: factors determining lutein accumulation in Muriellopsis sp. (Chlorophyta). J. Biotechnol. 76, 51-59 (2000).

6. Del Campo, J. A. et al. Accumulation of astaxanthin and lutein in Chlorella zofingiensis (Chlorophyta). Appl. Microbiol. Biotechnol. 64, 848-854 (2004).

7. Ip, P.-F., Wong, K.-H. \& Chen, F. Enhanced production of astaxanthin by the green microalga Chlorella zofingiensis in mixotrophic culture. Process Biochem. 39, 1761-1766 (2004).

8. Del Campo, J. A., García-González, M. \& Guerrero, M. G. Outdoor cultivation of microalgae for carotenoid production: current state and perspectives. Appl. Microbiol. Biotechnol. 74, 1163-1174 (2007).

9. Ip, P.-F. \& Chen, F. Production of astaxanthin by the green microalga Chlorella zofingiensis in the dark. Process Biochem. 40, 733-738 (2005).

10. Sharma, R. Effects of culture conditions on growth and biochemical profile of Chlorella vulgaris. J. Plant Pathol. Microbiol. 3, 100131 (2012).

11. Choi, H.-J. \& Lee, S.-Y. Advances in microalgal biomass/bioenergy production with agricultural by-products: analysis with various growth rate models. Environ. Eng. Res. 24, 271-278 (2018).

12. Sharma, A. K., Sahoo, P. K., Singhal, S. \& Patel, A. Impact of various media and organic carbon sources on biofuel production potential from Chlorella spp. 3 Biotech. 6, 116 (2016).

13. Xu, Y., Ibrahim, I. M. \& Harvey, P. J. The influence of photoperiod and light intensity on the growth and photosynthesis of Dunaliella salina (chlorophyta) CCAP 19/30. Plant Physiol. Biochem. 106, 305-315 (2016).

14. Singh, S. \& Singh, P. Effect of temperature and light on the growth of algae species: a review. Renew. Sustain. Energy Rev. 50, 431-444 (2015).

15. Gururani, M., Venkatesh, J. \& Tran, L. S. P. Regulation of photosynthesis during abiotic stress-induced photoinhibition. Mol. Plant 8, 1304-1320 (2015).

16. Hemalatha, A., Girija, K., Parthiban, C., Saranya, C. \& Anantharaman, P. Antioxidant properties and total phenolic content of a marine diatom, green microalgae. Adv. Appl. Sci. Res. 4, 115-157 (2013).

17. Mulders, K. J. M. et al. Nitrogen-depleted Chlorella zofingiensis produces astaxanthin, ketolutein and their fatty acid esters: a carotenoid metabolism study. J. Appl. Phycol. 27, 125-140 (2014).

18. Xiao, Y. et al. Photosynthetic accumulation of lutein in Auxenochlorella protothecoides after heterotrophic growth. Mar. Drugs 16, $283(2018)$.

19. Wan, L. et al. De novo transcriptomic analysis of an oleaginous microalga: pathway description and gene discovery for production of next-generation biofuels. PLoS ONE 7, e35142 (2012).

20. Carrier, G. et al. Comparative transcriptome of wild type and selected strains of the microalgae Tisochrysis lutea provides insights into the genetic basis, lipid metabolism and the life cycle. PLoS ONE 9, e86889 (2014).

21. Rismani-Yazdi, H., Haznedaroglu, B. Z., Bibby, K. \& Peccia, J. Transcriptome sequencing and annotation of the microalgae Dunaliella tertiolecta: pathway description and gene discovery for production of next-generation biofuels. BMC Genom. 12, 148 (2011).

22. Hovde, B. T. et al. Genomic characterization reveals significant divergence within Chlorella sorokiniana (Chlorellales, Trebouxiophyceae). Algal Res. 35, 449-461 (2018).

23. Malavasi, V. et al. Deep genomic analysis of the Chlorella sorokiniana SAG 211-8k chloroplast. Eur. J. Phycol. 52, 320-329 (2017). 
24. Lowe, R., Shirley, N., Bleackley, M., Dolan, S. \& Shafee, T. Transcriptomics technologies. PLOS Comput. Biol. 13, e1005457 (2017).

25. Guarnieri, M. T. et al. Examination of triacylglycerol biosynthetic pathways via de novo transcriptomic and proteomic analyses in an unsequenced microalga. PLoS ONE 6, e25851 (2011).

26. Li, Q., Liu, J., Zhang, L. \& Liu, Q. D. Novo transcriptome analysis of an aerial microalga Trentepohlia jolithus: pathway description and gene discovery for carbon fixation and carotenoid biosynthesis. PLoS ONE 9, e108488 (2014).

27. Gao, C. et al. Oil accumulation mechanisms of the oleaginous microalga Chlorella protothecoides revealed through its genome, transcriptomes, and proteomes. BMC Genom. 15, 582 (2014).

28. Sun, D. et al. De novo transcriptome profiling uncovers a drastic downregulation of photosynthesis upon nitrogen deprivation in the nonmodel green alga Botryosphaerella sudeticus. BMC Genom. 14, 715 (2013).

29. Chan, C. X. et al. Porphyra (Bangiophyceae) transcriptomes provide insights into red algal development and metabolism. J. Phycol. 48, 1328-1342 (2012).

30. He, Y., Ma, Y., Du, Y. \& Shen, S. Differential gene expression for carotenoid biosynthesis in a green alga Ulva prolifera based on transcriptome analysis. BMC Genom. 19, 916 (2018).

31. Azaman, S. N. A., Nagao, N., Yusoff, F. M., Tan, S. W. \& Yeap, S. K. A comparison of the morphological and biochemical characteristics of Chlorella sorokiniana and Chlorella zofingiensis cultured under photoautotrophic and mixotrophic conditions. Peer J. 5, e3473 (2017).

32. Grabherr, M. G. et al. Full-length transcriptome assembly from RNA-Seq data without a reference genome. Nat. Biotechnol. 29, 644-652 (2011).

33. Paniagua-Michel, J., Olmos-Soto, J. \& Ruiz, M. A. Microbial Carotenoids from Bacteria and Microalgae 1-12 (Springer, Berlin, 2012).

34. Ruiz-Sola, M. Á. \& Rodríguez-Concepción, M. Carotenoid biosynthesis in Arabidopsis: a colorful pathway. Arabidopsis Book 10, $\mathrm{e} 0158(2012)$.

35. Ahrazem, O., Gómez-Gómez, L., Rodrigo, M., Avalos, J. \& Limón, M. Carotenoid cleavage oxygenases from microbes and photosynthetic organisms: features and functions. Int. J. Mol. Sci. 17, 1781 (2016).

36. Cui, H., Wang, Y. \& Qin, S. Genomewide analysis of carotenoid cleavage dioxygenases in unicellular and filamentous cyanobacteria. Int J Genomics 2012, 164690 (2012).

37. Schmidt, A. et al. A bifunctional geranyl and geranylgeranyl diphosphate synthase is involved in terpene oleoresin formation in Picea abies. Plant Physiol. 152, 639-655 (2010).

38. Cagliari, A. et al. Biosynthesis of triacylglycerols (TAGs) in plants and algae. Int. J. Plant Biol. 2, 10 (2011).

39. Sasaki, Y. \& Nagano, Y. Plant acetyl-CoA carboxylase: structure, biosynthesis, regulation, and gene manipulation for plant breeding. Biosci. Biotechnol. Biochem. 68, 1175-1184 (2004).

40. Huang, W. et al. Transcriptome analysis of Chlorella zofingiensis to identify genes and their expressions involved in astaxanthin and triacylglycerol biosynthesis. Algal Res. 17, 236-243 (2016).

41. Wang, H., Fu, R. \& Pei, G. A study on lipid production of the mixotrophic microalgae Phaeodactylum tricornutum on various carbon sources. Afr. J. Microbiol. Res. 6, 1041-1047 (2012).

42. Zhan, J., Rong, J. \& Wang, Q. Mixotrophic cultivation, a preferable microalgae cultivation mode for biomass/bioenergy production, and bioremediation, advances and prospect. Int. J. Hydrog. Energy 42, 8505-8517 (2017).

43. Quinlan, R. F. et al. Synergistic interactions between carotene ring hydroxylases drive lutein formation in plant carotenoid biosynthesis. Plant Physiol. 160, 204-214 (2012).

44. Cecchin, M. et al. Molecular basis of autotrophic vs mixotrophic growth in Chlorella sorokiniana. Sci. Rep. 8, 6465 (2018).

45. Andre, C., Haslam, R. P. \& Shanklin, J. Feedback regulation of plastidic acetyl-CoA carboxylase by 18:1-acyl carrier protein in Brassica napus. Proc. Natl. Acad. Sci. 109, 10107-10112 (2012).

46. Sirikhachornkit, A., Suttangkakul, A., Vuttipongchaikij, S. \& Juntawong, P. De novo transcriptome analysis and gene expression profiling of an oleaginous microalga Scenedesmus acutus TISTR8540 during nitrogen deprivation-induced lipid accumulation. Sci. Rep. 8, 3668 (2018).

47. Alboresi, A. et al. Light remodels lipid biosynthesis in Nannochloropsis gaditana by modulating carbon partitioning between organelles. Plant Physiol. 171, 2468-2482 (2016).

48. Han, D. et al. Metabolic remodeling of membrane glycerolipids in the microalga Nannochloropsis oceanica under nitrogen deprivation. Front. Mar. Sci. 4, 242 (2017).

49. Fan, J. et al. Genomic foundation of starch to lipid switch in oleaginous Chlorella spp. Plant Physiol. 169, 2444-2461 (2015).

50. Work, V. H. et al. Increased lipid accumulation in the Chlamydomonas reinhardtii sta7-10 starchless isoamylase mutant and increased carbohydrate synthesis in complemented strains. Eukaryot. Cell 9, 1251-1261 (2010).

51. Sirikhachornkit, A. et al. Increasing the triacylglycerol content in Dunaliella tertiolecta through isolation of starch-deficient mutants. J. Microbiol. Biotechnol. 26, 854-866 (2016).

52. De Jaeger, L. et al. Superior triacylglycerol (TAG) accumulation in starchless mutants of Scenedesmus obliquus: (I) mutant generation and characterization. Biotechnol. Biofuels 7, 69 (2014).

53. Ratnapuram, H. P., Vutukuru, S. \& Yadavalli, R. Mixotrophic transition induced lipid productivity in Chlorella pyrenoidosa under stress conditions for biodiesel production. Heliyon 4, e00496 (2018).

54. Wang, Z. T., Ullrich, N., Joo, S., Waffenschmidt, S. \& Goodenough, U. Algal lipid bodies: stress induction, purification, and biochemical characterization in wild-type and starchless Chlamydomonas reinhardtii. Eukaryot. Cell 8, 1856-1868 (2009).

55. Li, Y., Han, D., Hu, G., Sommerfeld, M. \& Hu, Q. Inhibition of starch synthesis results in overproduction of lipids in Chlamydomonas reinhardtii. Biotechnol. Bioeng. 107, 258-268 (2010).

56. Li, Y., Fei, X. \& Deng, X. Novel molecular insights into nitrogen starvation-induced triacylglycerols accumulation revealed by differential gene expression analysis in green algae Micractinium pusillum. Biomass Bioenerg. 42, 199-211 (2012).

57. Stanke, M. et al. AUGUSTUS: ab initio prediction of alternative transcripts. Nucleic Acids Res. 34, W435-W439 (2006).

58. Kanehisa, M. \& Goto, S. KEGG: kyoto encyclopedia of genes and genomes. Nucleic Acids Res. 28, 27-30 (2000).

59. Lohse, M. et al. Mercator: a fast and simple web server for genome scale functional annotation of plant sequence data. Plant Cell Environ. 37, 1250-1258 (2014).

60. Liao, Y., Smyth, G. K. \& Shi, W. The subread aligner: fast, accurate and scalable read mapping by seed-and-vote. Nucleic Acids Res. 41, e108-e108 (2013).

61. Liao, Y., Smyth, G. K. \& Shi, W. featureCounts: an efficient general purpose program for assigning sequence reads to genomic features. Bioinformatics 30, 923-930 (2014).

62. Love, M. I., Huber, W. \& Anders, S. Moderated estimation of fold change and dispersion for RNA-seq data with DESeq2. Genome Biol. 15, 550 (2014).

63. Hornik, K. The R FAQ. https://CRAN.R-project.org/doc/FAQ/R-FAQ.html (2020). Accessed 1 May 2020.

64. Giorgi, F. M., Del Fabbro, C. \& Licausi, F. Comparative study of RNA-seq- and microarray-derived coexpression networks in Arabidopsis thaliana. Bioinformatics 29, 717-724 (2013).

65. Li, L., Zhang, G. \& Wang, Q. De novo transcriptomic analysis of Chlorella sorokiniana reveals differential genes expression in photosynthetic carbon fixation and lipid production. BMC Microbiol. 16, 223 (2016). 


\section{Acknowledgements}

We would like to thank Ms Boo Sok Yee, Ms Lily Boo and Ms Norlaily Mohd Ali, Ms Nancy Liew Woan Charn and Dr Hyunh Ky for their technical support and guidance on analyzing the data. The study was funded by Xiamen University Malaysia (Xiamen University Malaysia Research Fund; Grant no. XMUMRF/2018-C1/ ICAM/0002) and Institute of Bioscience, Higher Institution Centre of Excellence (IBS HICoE) grant from the Ministry of Higher Education, Government of Malaysia (Vote No. 6369100).

\section{Author contributions}

S.N.A.A, Y.S.K, D.C.J.W: Conceptualization. S.N.A.A., D.C.J.W, T.S.W., Y.S.K.: Investigation; S.N.A.A., D.C.J.W.: writing-original draft preparation; Y.S.K., T.S.W., F.M.Y, N.N.: writing-review and editing; Y.S.K., T.S.W., F.M.Y, N.N.: supervision; Y.S.K. and F.M.Y.: funding acquisition.

\section{Competing interests}

The authors declare no competing interests.

\section{Additional information \\ Supplementary information is available for this paper at https://doi.org/10.1038/s41598-020-74410-4.}

Correspondence and requests for materials should be addressed to S.K.Y.

Reprints and permissions information is available at www.nature.com/reprints.

Publisher's note Springer Nature remains neutral with regard to jurisdictional claims in published maps and institutional affiliations.

(c) (1) Open Access This article is licensed under a Creative Commons Attribution 4.0 International License, which permits use, sharing, adaptation, distribution and reproduction in any medium or format, as long as you give appropriate credit to the original author(s) and the source, provide a link to the Creative Commons licence, and indicate if changes were made. The images or other third party material in this article are included in the article's Creative Commons licence, unless indicated otherwise in a credit line to the material. If material is not included in the article's Creative Commons licence and your intended use is not permitted by statutory regulation or exceeds the permitted use, you will need to obtain permission directly from the copyright holder. To view a copy of this licence, visit http://creativecommons.org/licenses/by/4.0/.

(C) The Author(s) 2020 